\title{
A Simple Homogenized Model for the Non-Linear Analysis of FRP Strengthened Masonry Structures
}

\author{
G. Milani ${ }^{1}$ and P.B. Lourenço ${ }^{2}$ \\ ${ }^{1}$ Technical University of Milan, Italy \\ ${ }^{2}$ University of Minho, Guimarães, Portugal
}

\begin{abstract}
A two-step model able to predict the non-linear response of FRP strengthened threedimensional masonry structures is presented. In the first step, non-strengthened masonry is substituted by a macroscopically equivalent homogeneous material through a kinematic model based on finite elements and working on a heterogeneous assemblage of blocks. Non-linearity is concentrated exclusively on joints reduced to interfaces, exhibiting a frictional behaviour with limited tensile and compressive strength with softening. The homogenized stress-strain behaviour evaluated at the meso-scale is then implemented at a structural level in a finite element non-linear code, relying on an assemblage of rigid infinitely resistant six-noded wedge elements and non-linear interfaces, with deterioration of the mechanical properties. FRP reinforcing strips are modelled through rigid triangles and non-linear interfaces between adjoining triangles. Delamination from the support is accounted for, by modelling FRP-masonry bond by means of non-linear softening triangular interfaces. Italian code CNR DT 2000 formulas are used to evaluate peak interface tangential strength and post peak behaviour. A structural examples relying into a masonry deep beam is presented for validation purposes.
\end{abstract}

Keywords: masonry, fibre-reinforced polymer, non-linear model, homogenization, finite element method.

\section{Introduction}

The foreseen inadequate performance of masonry structures under earthquakes, particularly in the case of old buildings or inadequate modern construction, is a common issue in many countries worldwide and is essentially due to the mortar joints weakness. Conventional retrofitting, such as external reinforcement with steel plates or reinforced concrete overlay, has proven to be impractical, expensive and add considerable mass to the structure (which may increase earthquake-induced 
inertia forces). In this context, the utilization of externally bonded FRP strips seems an interesting solution due to the limited invasiveness, durability of the FRP and good performance at failure.

While FRP external reinforcement is now very popular, the prediction of its mechanical behaviour when bonded to masonry in the inelastic range still remains a difficult task. Several concurring factors make the analysis of strengthened masonry structures very challenging. Among others, the most important are: (1) the heterogeneity of the masonry material, (2) the brittle behaviour in tension of mortar joints, even at very low levels of external loads, (3) the delamination of the FRP from the support, which is typically brittle, and (4) the complex interaction between flexural strength and vertical pre-compression in case of bending.

At present, it is the authors' opinion that an efficient analysis of both FRP strengthened and non-strengthened large scale masonry structures in the non-linear range requires a micro-macro or macro-computational approach 0-[8]. Indeed, a numerical model to use at structural level should be sufficiently simple, reliable and efficient to allow the fast evaluation of (a) collapse loads, (b) displacements near collapse, (c) failure mechanism, and (d) post peak behaviour of the structures.

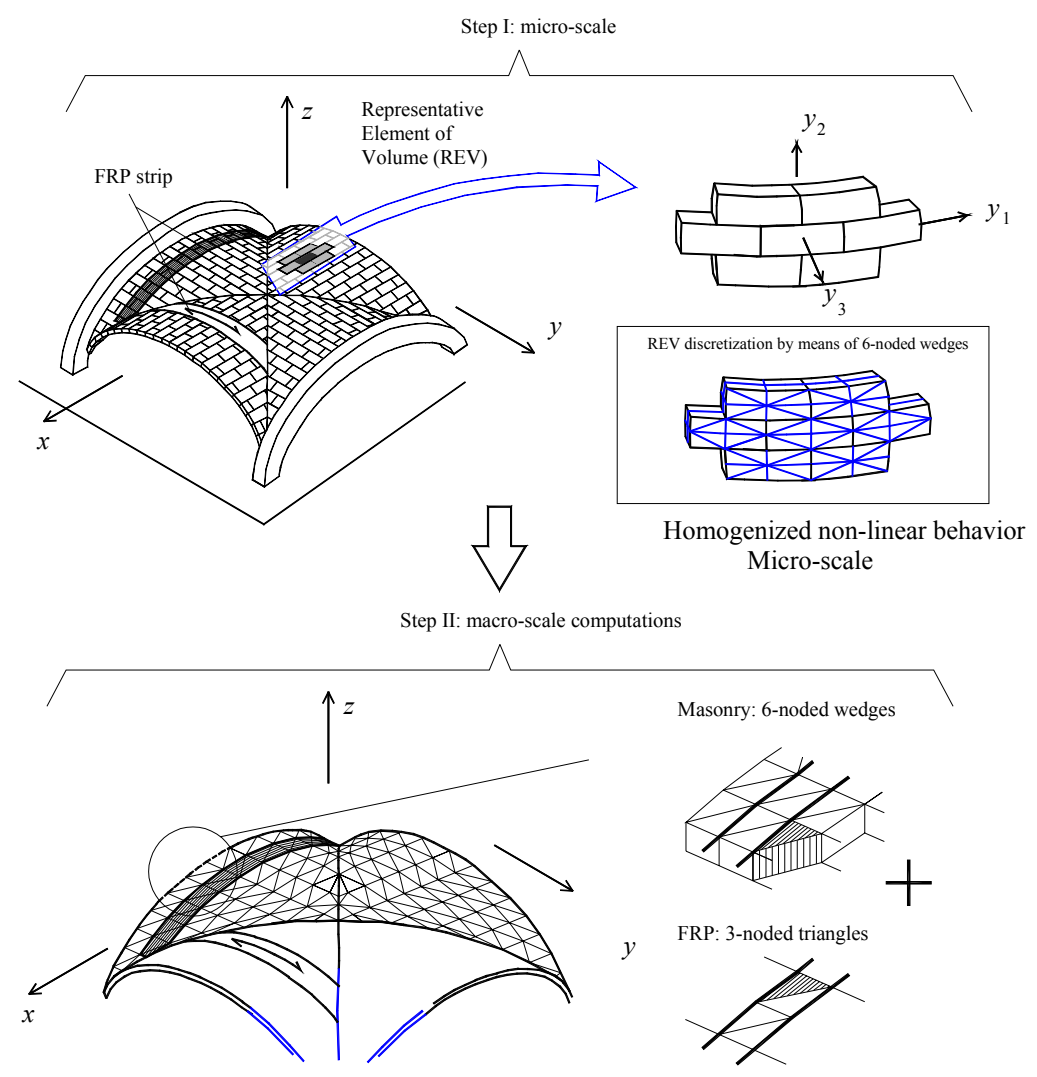

Figure 1: Two-step kinematic simplifying homogenization approach. Identification of a Representative Element of Volume (REV), subsequent evaluation of the nonlinear non-strengthened macroscopic behaviour of the REV, implementation at a structural level within a non-linear FE code (non-linear behaviour of homogenized masonry and FRP). 
In this paper, a simple two-step micro-macro model is used to analyze efficiently masonry FRP strengthened structures, see

Figure 1. In the first step, hereafter called meso-modelling, masonry is substituted by a macroscopic equivalent material through the application of a simplified averaging procedure, in which a REV constituted by a central brick interconnected with its six neighbors through zero thickness joints is meshed with six-noded wedges and non-linear softening interfaces (mortar joints and brick-brick interfaces that allow potential internal cracks in the bricks). The approach allows estimating in an approximate way masonry macroscopic non-linear behaviour under in- and outof-plane loads, at different orientations of the actions with respect to material axes.

In the second step, full masonry structures are analyzed in the non-linear range through a tailored FE non-linear code specifically developed to conduct reliable and simple analyses on structures with any shape and under general loading conditions. Six-noded rigid and infinitely resistant wedges are utilized, e.g. [9][10], with elastic and inelastic deformation allowed only at the interfaces between adjoining elements. Only the knowledge of masonry orthotropic stress-strain relations, i.e. information provided at the meso-scale, is therefore required.

FRP strips are modelled by means of triangular rigid elements and possible elastic and inelastic deformation is allowed only at the linear interfaces between contiguous triangles. Masonry and FRP layers interact by means of interfacial tangential actions between triangles (FRP) and wedges (masonry). There, to properly account for the detachment of the strip from the support, an elastodamaging shear stress-slip relationship is assumed, in agreement with codes of practice formulas dealing with delamination (e.g. Italian CNR DT-200 0).

In order to circumvent some typical drawbacks of standard FEs when dealing with softening materials, a sequential quadratic programming approach (SQP) is adopted to solve the global non-linear problem. The algorithm is finally tested on a medium size deep beam reinforced with diagonal and horizontal FRP strips.

\section{Unreinforced masonry: simplified homogenization}

The homogenization proposed pertains to running bond non-strengthened masonry, regarded as an assemblage of bricks interacting through interfaces (mortar joints). Bricks are supposed infinitely resistant, whereas for joints a Mohr Coulomb failure criterion with tension cut-off and compressive limited strength is adopted. In this way, a full description of the model can be given at a micro-scale considering a representative volume constituted by a generic brick interacting with its six neighbors. A sub-class of possible elementary deformation modes acting in the unit cell is a priori chosen in order to describe joints cracking under normal, tangential actions and bending. Then, a numerical procedure of identification between the $3 \mathrm{D}$ discrete system and a continuum $2 \mathrm{D}$ equivalent model is proposed, equating the internal work in the two models.

In the heterogeneous model, the whole REV is meshed through six-noded wedge elements interconnected by interfaces (internal brick-brick interfaces and mortar joints, see Figure 2-a). The motion of a generic element $E$, see Figure 2, is 
described as a function of its centroid $\left(C^{E}\right)$ displacement vector $\mathbf{u}^{E}$ (components $u_{x x}^{E}, u_{y y}^{E}$ and $u_{z z}^{E}$ ) and of its rotation vector $\boldsymbol{\Phi}^{E}$ (components $\Phi_{x x}^{E}, \Phi_{y y}^{E}$ and $\Phi_{z z}^{E}$ ) around centroid.

When two contiguous bricks $M$ and $N$ are considered, the displacement of a generic point $P$ in a position $\xi \in \Gamma_{12}$ belonging respectively to $M$ and $N$ (where $\Gamma_{12}$ indicates the common interface between the two elements) is:

$$
\begin{aligned}
& \mathbf{u}^{M}(P)=\mathbf{u}^{M}+\mathbf{M}\left(\boldsymbol{\Phi}^{M}\right)\left(P-C^{M}\right) \\
& \mathbf{u}^{N}(P)=\mathbf{u}^{N}+\mathbf{M}\left(\boldsymbol{\Phi}^{N}\right)\left(P-C^{N}\right)
\end{aligned}
$$

Where $\mathbf{M}(\boldsymbol{\Phi})=\left[\begin{array}{ccc}0 & -\Phi_{z z} & \Phi_{y y} \\ \Phi_{z z} & 0 & -\Phi_{x x} \\ -\Phi_{y y} & \Phi_{x x} & 0\end{array}\right]$

In equation ( 1 ) the position $\xi$ of point $P$ is evaluated with reference to a local frame of reference $\left(\begin{array}{ll}\xi_{1} & \xi_{2}\end{array}\right)$ with origin on the centroid on the interface, Figure 2-b. Jump of displacements $[\mathbf{U}(P)]$ between bricks $M$ and $N$ in a point $\xi \in \Gamma_{12}$ is expressed by:

$$
\lfloor\mathbf{U}(P)\rfloor=\mathbf{u}^{M}(P)-\mathbf{u}^{N}(P)=\mathbf{u}^{M}-\mathbf{u}^{N}+\mathbf{M}\left(\boldsymbol{\Phi}^{M}\right)\left(P-C^{M}\right)-\mathbf{M}\left(\Phi^{N}\right)\left(P-C^{N}\right)
$$

Having defined a local frame of reference $\xi_{1}-\xi_{2}-\xi_{3}$ for the interface between $N$ and $M$ elements (vertices corresponding to nodes $P_{1}, P_{2}, P_{4}$ and $P_{5}$, Figure 2-b and -c), we assume that it is characterized by two axes $\left(\mathbf{e}_{1}-\mathbf{e}_{2}\right)$ laying on the interface plane and mutually orthogonal, while the third perpendicular axis to the interface is $\mathbf{e}_{3}$. Thus, unitary vectors $\mathbf{e}_{1}-\mathbf{e}_{2}-\mathbf{e}_{3}$ may be expressed in the global coordinate system as $\mathbf{e}_{1}=\mathbf{e}_{2} \times \mathbf{e}_{3}, \mathbf{e}_{2}=\frac{P_{2}-P_{1}}{\left\|P_{2}-P_{1}\right\|}$ and $\mathbf{e}_{3}=\widetilde{\mathbf{e}}_{1} \times \mathbf{e}_{2}$ with $\widetilde{\mathbf{e}}_{1}=\frac{P_{4}-P_{1}}{\left\|P_{4}-P_{1}\right\|}$.

The rotation matrix $\mathbf{R}_{\mathfrak{e}}$, with respect to the global coordinate system jump of displacements, may be written in the local system as:

$$
[\widetilde{\mathbf{U}}(P)]=\mathbf{R}_{\mathbf{e}}[\mathbf{U}(P)]
$$

where the superscript $\sim$ indicates quantities evaluated in the local system.

From ( 3 ), it is possible to evaluate the work dissipated on $\Gamma_{12}$ as follows:

$$
\pi=\int_{I}\left[\boldsymbol{\sigma}_{M}(P) \cdot \widetilde{\mathbf{U}}_{M}(P)+\boldsymbol{\sigma}_{N}(P) \cdot \widetilde{\mathbf{U}}_{N}(P)\right] d S=\int_{I} \boldsymbol{\sigma}_{M}(P) \cdot[\widetilde{\mathbf{U}}(P)] d S
$$


Where $\boldsymbol{\sigma}_{M}(P)=\left[\begin{array}{lll}\tau_{13}(P) & \tau_{23}(P) & \sigma_{33}(P)\end{array}\right]^{T}$ is the stress vector acting at $P$ on element $M$, with $\boldsymbol{\sigma}_{N}(P)=-\boldsymbol{\sigma}_{M}(P)$.

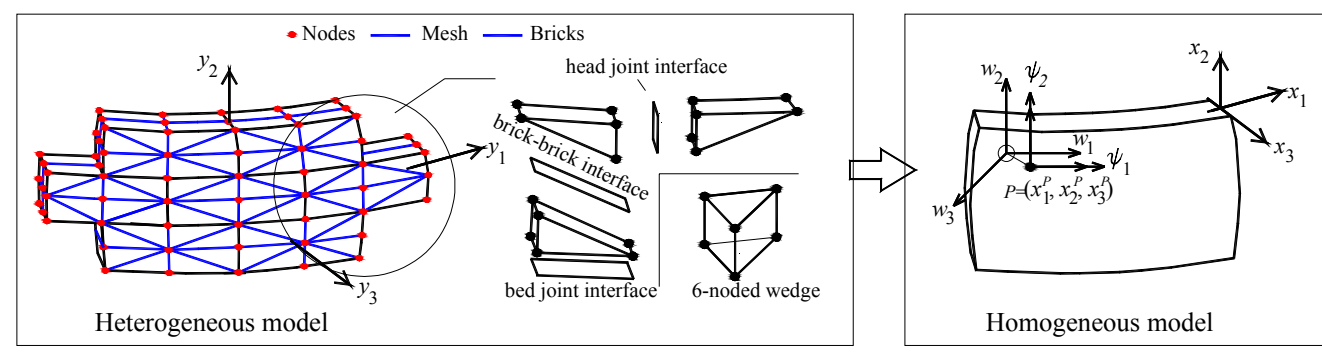

$-\mathrm{a}$

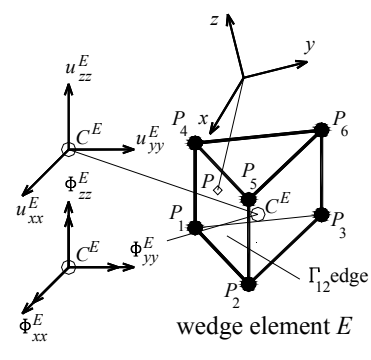

$-b$

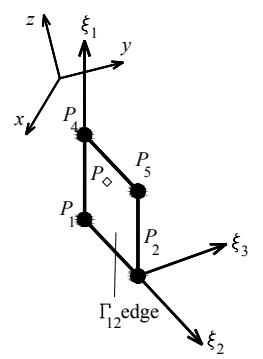

$-\mathrm{c}$
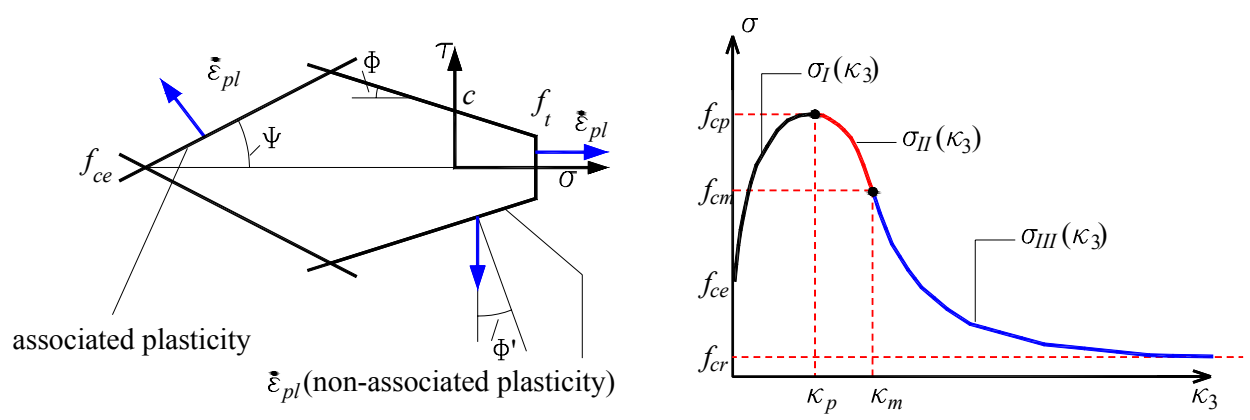

$-d$

Figure 2: -a: FE discretization of the non-strengthened REV. - b: Rigid infinitely resistant six-noded wedge element used for the REV discretization.-c: $\Gamma_{12}$ interface between contiguous elements. $-\mathrm{d}$ : Modified Mohr-Coulomb criterion for the mortar joint reduced to interface (left) and hardening/softening law in compression (right) as a function of the inelastic parameter $\kappa_{3}$

When dealing with the continuous model, a standard Cauchy bi-dimensional continuum, Figure 2-a, is considered. Here the global frame of reference is identified by the vectors $x_{1}, x_{2}$ and $x_{3}$. 
The displacement field of a point $\mathbf{P}$ (coordinates $\left[\begin{array}{lll}x_{1}^{P} & x_{2}^{P} & x_{3}^{P}\end{array}\right]$ ) belonging to the equivalent continuum plate is given by fields $\mathbf{w}(\mathbf{x})$ (components $w_{1}, w_{2}$ and $w_{3}$ ) and $\Psi(\mathbf{x})$ (components $\Psi_{1}$ and $\Psi_{2}$ ), representing respectively the displacements and rotations of the plate in correspondence of the point $\mathbf{x}=\left[\begin{array}{lll}x_{1}^{P} & x_{2}^{P} & 0\end{array}\right]$ laying in the middle plane of the continuum regarded as a plate (i.e. with two dimensions much bigger than a third one, the thickness).

For in- and out-of-plane loads, membrane forces vector $\mathrm{N}$ (components $N_{11}, N_{12}$ and $N_{22}$ ), moments $\mathrm{M}$ (bending $M_{11}, M_{22}$ and torsion $M_{12}$ ) and out-of-plane shear T (components $T_{13}$ and $T_{23}$ ) contribute to the internal work. In particular, the work dissipated by an equivalent plate model is simply:

$$
\pi=\left[\begin{array}{lll}
N_{11} & N_{12} & N_{22}
\end{array}\right]\left[\begin{array}{c}
E_{11} \\
E_{12}+E_{21} \\
E_{22}
\end{array}\right]+\left[\begin{array}{ll}
T_{13} & T_{23}
\end{array}\right]\left[\begin{array}{l}
\gamma_{13} \\
\gamma_{23}
\end{array}\right]+\left[\begin{array}{lll}
M_{11} & M_{12} & M_{22}
\end{array}\right]\left[\begin{array}{c}
\chi_{11} \\
\chi_{12}+\chi_{21} \\
\chi_{22}
\end{array}\right]
$$

where $\mathbf{E}$ is the in-plane strain vector, $\chi$ the out-of-plane strain vector and $\gamma$ the outof-plane shear strain.

\subsection{Homogeneous model}

To substitute the heterogeneous material with the homogeneous equivalent $2 \mathrm{D}$ model, a simple compatible identification model is proposed [11] [12], where the work by the blocks model, equation ( 4 ), is equated to the work ( 5 ) by the equivalent model.

At this aim, fields $\mathbf{w}(\mathbf{x})$ and $\boldsymbol{\Psi}(\mathbf{x})$ are a priori chosen as a combination of elementary deformations in the unit cell, corresponding to actual failure mechanisms occurring, according to experimental evidences, in presence of running bond brickwork with weak joints reduced to interfaces. From a practical point of view, fields $\mathbf{w}(\mathbf{x})$ and $\boldsymbol{\Psi}(\mathbf{x})$ corresponding to each sub-class of regular motions are obtained assuming alternatively one component of vector $\mathbf{E}, \gamma$ or $\chi$ unitary and setting all the other components equal to zero, subsequently choosing the most simple polynomial expressions for $\mathbf{w}(\mathbf{x})$ and $\Psi(\mathbf{x})$ which comply with the compatibility equations. Once fields $\mathbf{w}(\mathbf{x})$ and $\Psi(\mathbf{x})$ are known from the procedure described, rotations and displacements of each element belonging to the REV in the heterogeneous model are determined solving a boundary value problem on the REV where displacements (or displacement increments) on the boundary are imposed.

Since the aim of this paper is to model the strengthening effect induced by FRP in bending, at the macro-scale homogenized three dimensional wedge-shaped elements are used for masonry (see following sections and

Figure 1). Consequently, non-strengthened brickwork behaviour in flexion is obtained by integration of in plane actions at a structural level (step II). 
Therefore, at the micro-scale it is possible to limit the study to in-plane and outof-plane shear actions ( $\mathbf{E}, \boldsymbol{\gamma}$ respectively).

For a generic brick-brick or mortar interface, the elastic domain is, in the most general case, bounded by a composite yield surface that includes tension, shear and compression failure with softening, Figure 2-d. A multi-surface plasticity model is adopted, with softening in both tension and compression. The elastic domain is defined by each $\mathrm{i}$-th yield function $f_{i} \leq 0$, in the form $f_{i}\left(\sigma, \tau, \kappa_{i}\right)=\Phi_{i}(\sigma, \tau)+\Psi_{i}\left(\kappa_{i}\right)$, where scalar $\kappa_{i}$ rules the amount of softening of the i-th yield surface and $\Phi_{i}$ and $\Psi_{i}$ are generic functions representing respectively the initial i-th yield surface and the correction which accounts for the evolution of the strength during the inelastic deformation process. Total strain rate $\dot{\boldsymbol{\varepsilon}}$ is decomposed into an elastic component $\dot{\boldsymbol{\varepsilon}}_{e l}$ and a plastic component $\dot{\boldsymbol{\varepsilon}}_{p l}$. The elastic strain rate is related to the stress rate by the elastic constitutive matrix $\mathbf{D}$ as $\dot{\boldsymbol{\sigma}}=\mathbf{D} \dot{\boldsymbol{\varepsilon}}_{e l}$, whereas the non-associated plasticity reads as $\dot{\boldsymbol{\varepsilon}}_{p l}=\lambda_{i} \frac{\partial g_{i}}{\partial \boldsymbol{\sigma}}$, where $g_{i}$ is the plastic potential corresponding to the i-th yield surface (which rules the direction of $\dot{\boldsymbol{\varepsilon}}_{p l}$ in the stress space) and $\boldsymbol{\sigma}=\left[\begin{array}{ll}\sigma & \tau\end{array}\right]^{T}$.

The multi-surface plasticity model adopted is the classical Mohr-Coulomb type strength criterion, with a tension cut-off and a linear compression cap, Figure 2-d. ft and $\mathrm{fc}$ are, respectively, tensile and compressive Mode-I strength, $\mathrm{c}$ is the cohesion, $\Phi$ is the friction angle, and $\Psi$ is the angle which defines the linear compression cap. For the tension mode, exponential softening is assumed, i.e. $f_{1}\left(\boldsymbol{\sigma}, \kappa_{1}\right)=\sigma-f_{t}\left(\kappa_{1}\right)$. where $f_{t}\left(\kappa_{1}\right)$ deteriorates in agreement with the following formula:

$$
f_{t}\left(\kappa_{1}\right)=f_{t 0} e^{-\frac{f_{t 0}}{G_{f}^{I}} \kappa_{1}}
$$

being $f_{t 0}$ the initial joint tensile strength and $G_{f}^{I}$ the mode I fracture energy. An associated flow rule is assumed. For the shear mode, the Mohr-Coulomb yield function reads $f_{2}\left(\boldsymbol{\sigma}, \kappa_{2}\right)=|\tau|+\sigma \tan \phi\left(\kappa_{2}\right)-c\left(\kappa_{2}\right)$, where the yield values $c$ and $\tan \phi$ are ruled by the following formulas:

$$
\begin{aligned}
& c\left(\kappa_{2}\right)=c_{0} e^{-\frac{c_{0}}{G_{f}^{I I}} \kappa_{2}} \\
& \tan \phi=\tan \phi_{0}+\left(\tan \phi_{r}-\tan \phi_{0}\right)\left(c_{0}-c\right) / c_{0}
\end{aligned}
$$

being $c_{0}$ and $\tan \phi_{0}$ the initial cohesion and friction angle, $G_{f}^{I I}$ the mode II fracture energy and $\tan \phi_{r}$ the residual friction angle, here kept always equal to $75 \%$ of the initial one. A non-associated flow rule is assumed here, with zero dilatancy.

When dealing with the linearized compressive cap inelastic behaviour, a three function model [13], is utilized as shown in Figure 2-d, where the subscripts e, m, p 
and $\mathrm{r}$ of the yield value $f_{c}$ denote respectively, the elastic limit, medium, peak and residual values.

\begin{tabular}{|c|c|c|c|c|}
\hline & joint & $\begin{array}{c}\text { brick-brick } \\
\text { interface }\end{array}$ & & \\
\hline$E$ & $700^{(*)}$ & 1600 & {$[\mathrm{MPa}]$} & Young Modulus \\
\hline$G$ & $350^{(*)}$ & 800 & [MPa] & Shear Modulus \\
\hline$c$ & $1.4 f_{t}$ & 2 & {$[\mathrm{MPa}]$} & Cohesion \\
\hline$f_{t}$ & 0.2 & - & {$[\mathrm{MPa}]$} & Tensile strength \\
\hline$f_{c e}$ & $1 / 3 f_{c p}$ & - & {$[\mathrm{MPa}]$} & \multirow{6}{*}{$\begin{array}{l}\text { Compressive } \\
\text { hardening/softening } \\
\text { behaviour }\end{array}$} \\
\hline$f_{c p}$ & 7.5 & - & [MPa] & \\
\hline$f_{c m}$ & $0.8 f_{c p}$ & - & {$[\mathrm{MPa}]$} & \\
\hline$f_{c r}$ & $0.5 f_{c p}$ & - & {$[\mathrm{MPa}]$} & \\
\hline$\kappa_{p} / e_{h}$ & $5 \varepsilon_{e l}$ & - & {$[-]$} & \\
\hline$\kappa_{m} / e_{h}$ & $10 \varepsilon_{e l}$ & - & {$[-]$} & \\
\hline$\Phi$ & 25 & 45 & {$\left[{ }^{\circ}\right]$} & Friction angle \\
\hline$\Psi$ & 45 & - & {$\left[{ }^{\circ}\right]$} & $\begin{array}{l}\text { Angle of the } \\
\text { linearized } \\
\text { compressive cap }\end{array}$ \\
\hline$G_{f}^{I}$ & $\begin{array}{c}0.02(\text { Case } A) \\
0.2(\text { Case B })\end{array}$ & 10 & {$[\mathrm{~N} / \mathrm{mm}]$} & $\begin{array}{l}\text { Mode I fracture } \\
\text { energy }\end{array}$ \\
\hline$G_{f}^{I I}$ & $\begin{array}{c}0.01(\text { Case A) } \\
0.1(\text { Case B })\end{array}$ & 10 & {$[\mathrm{~N} / \mathrm{mm}]$} & $\begin{array}{l}\text { Mode II fracture } \\
\text { energy }\end{array}$ \\
\hline \multicolumn{5}{|c|}{$\begin{array}{l}\left(^{*} \text { Interface stiffness is evaluated as } \mathrm{E}^{*}(\mathrm{~V} 1+\mathrm{V} 2) /(4 \mathrm{~A}) \text {, with } \mathrm{V} 1 \text { and } \mathrm{V} 2 \text { being the }\right. \\
\text { volumes of the elements sharing the common interface under study and A being the } \\
\text { interface area }\end{array}$} \\
\hline
\end{tabular}

Table 1: Masonry deep beam. Mechanical properties adopted for constituent materials.

\subsection{Numerical simulations at a cell level}

This section provides an insight into the inelastic behaviour of masonry REVs with any shape, provided by the two-step model proposed.

To this aim, a running bond elementary cell constituted by $1 / 4$ of common solid clay Italian bricks (dimensions $62.5 \times 30 \times 14 \mathrm{~mm}$ ) is considered and is utilized at a structural level [8]. Elastic and inelastic material properties are summarized in Table 1. Two different values of fracture energy $G_{I}$ are assumed, the first corresponding realistically to existing masonry (Case A), the second assuming an almost perfect plastic behaviour in tension (Case B). FE discretization adopted is sketched in Figure 3-a. The behaviour in uniaxial tension is depicted in Figure 4-a for horizontal and vertical tension. The anisotropy of the homogenized model is particularly evident and is mainly due to the contribution in horizontal tension of the bed joint, which fails in shear. In order to validate the results, the curves obtained using classic 
FE simulation performed on a mesh with 384 elastic plane stress quadrilateral elements and mortar elasto-plastic interfaces are also represented, indicated as "FEM refined mesh" [14][15].

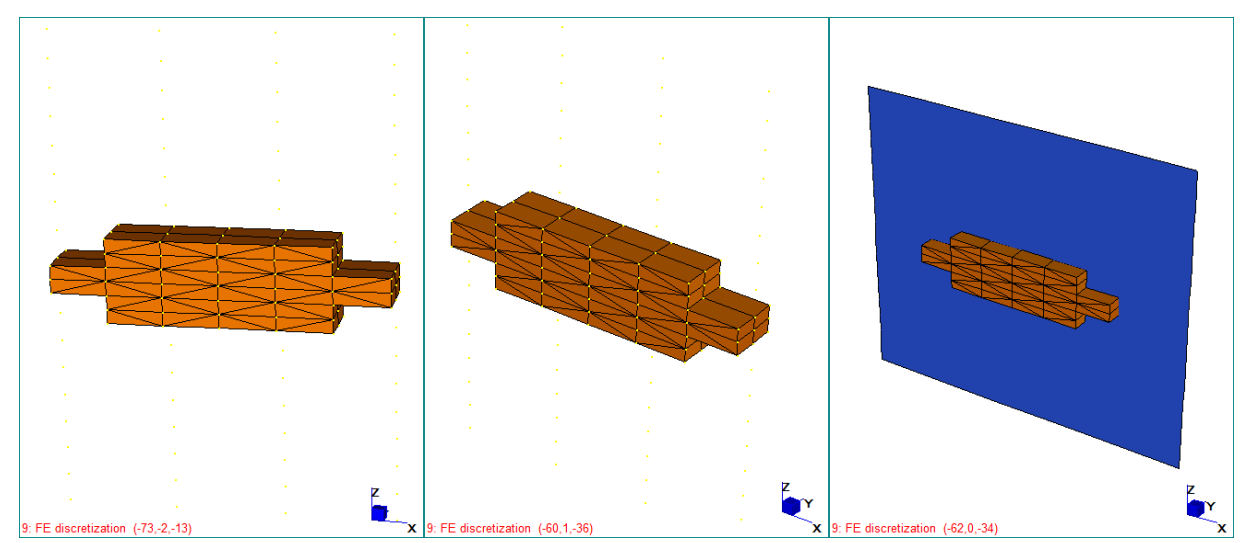

Figure 3: Masonry deep beam flat panel (-a) and circular arch (-b). Representative element of volume adopted for the simulations and FE discretization

As it is possible to notice, the agreement is almost perfect, even in the softening range. This is not surprising because fracture lines concentrates on joints reduced to interfaces, as demonstrated by the REV deformed shape depicted in Figure 4-b, where normal stress-shear masonry interfaces damage maps are also reported for the sake of completeness. A very similar behaviour is experienced in horizontal bending, as can be noted by the deformed shape and interfaces damage patch reported in Figure 4-c. For compression loads, the anisotropy is less evident, due to the low shear strength of the joint when compared to the compressive strength. Hence, little differences are expected when comparing the horizontal and vertical compression.

\section{Structural level}

The kinematic meso-scale model proposed allows obtaining masonry stress-strain diagrams at different orientations of load with respect to material axis. The out-ofplane behaviour may be reproduced as well. However, since six-noded wedge elements are used at a structural level, interfaces are subdivided into small rectangular areas and macroscopic internal actions $\mathrm{N}, \mathrm{T}, \mathrm{M}$ are obtained by integration of stress-strain curves evaluated on the REV. For this reason, only the average membrane behaviour (normal stress-normal strain and two mutually orthogonal tangential stress-tangential deformation curves for each interface) is required at the macro-scale.

At a structural level, rigid triangular shell elements are used to model FRP, Figure 1. Being rigid, elastic and inelastic deformation is allowed only at the interfaces between contiguous elements. It is also assumed that FRP may fail for pure axial action, when a stress threshold level is exceeded.

Delamination from the support is considered as well, since FRP triangular elements and masonry substrate are interconnected by means of triangular interfaces with linear jump of displacements acting normal and parallel to the interface. For the 
interface jump of displacements, the bilinear CNR DT 2000 softening law is assumed in the model, whereas for actions normal to the interface a perfectly bonded behaviour is assumed.
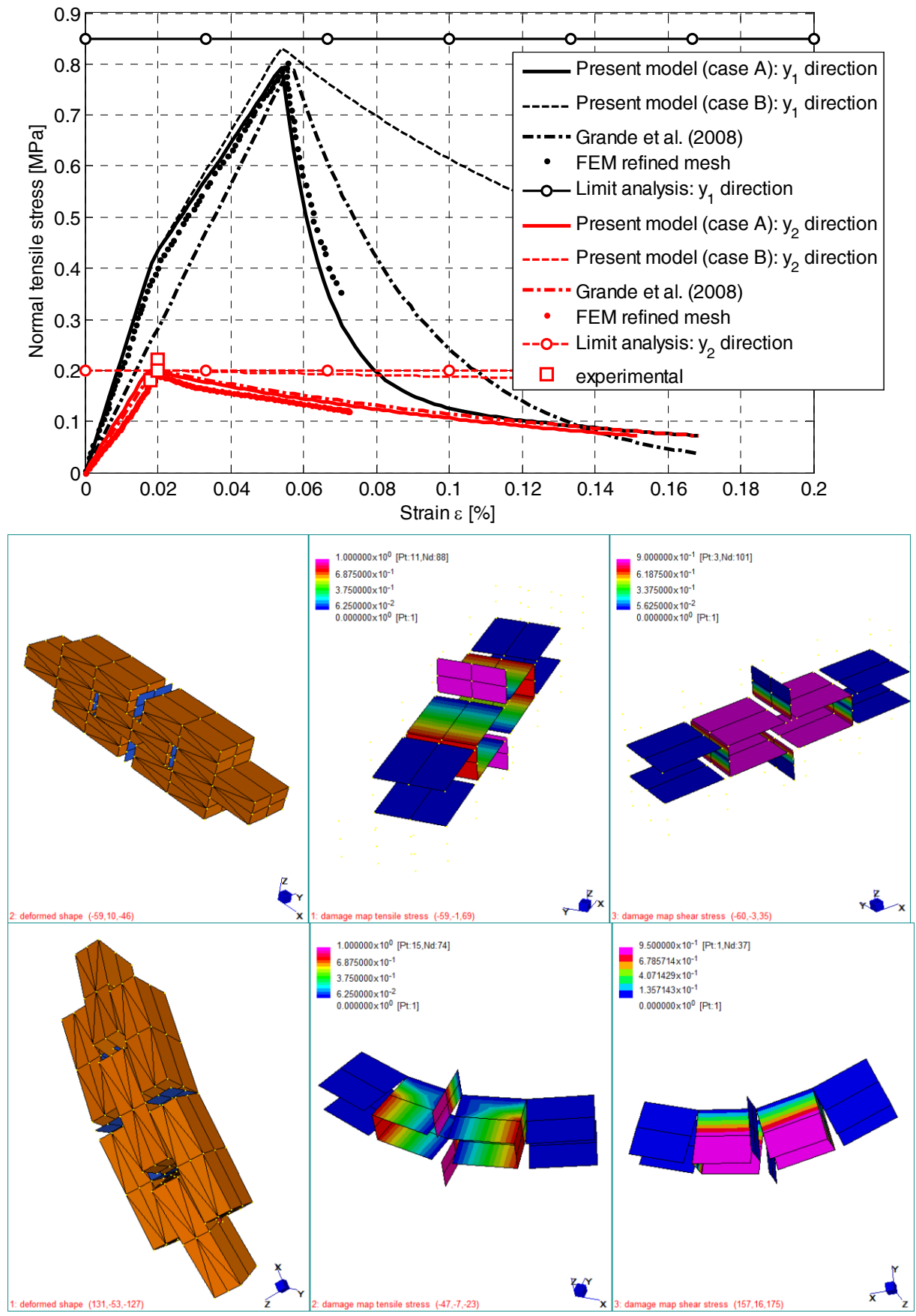

Figure 4: Masonry deep beam -a: Uniaxial response of the homogenization model along horizontal and vertical tension for two values of fracture energy. -b: REV deformed shape at collapse for horizontal tension (mesh used and magnified view) with indication of interface damage in horizontal tension (center) and vertical tension (right). -c: same as previous, but for horizontal bending. 
To summarize, the structural model is thus constituted by quadrilateral masonrymasonry interfaces, triangular FRP-masonry interfaces and FRP-FRP linear interfaces. All of them may potentially exhibit softening. At a structural level, to limit variables to a great extent, it is assumed to substitute the actual $2 \mathrm{D}$ behaviour of each interface by means of bending, tangential and normal non-linear springs, as illustrated in detail in [13]. In order to use a quadratic programming procedure to solve the problem at hand, each spring non-linear behaviour is approximated by means of a linear piece-wise constant approximation, as illustrated in detail in [10][11]. Such an approximation allows considering, in each single step, the structure as an assemblage constituted by rigid bodies and elastic-perfectly plastic springs.

Within this hypothesis, the elastic plastic response of a structure subjected to given proportionally increased loads is given by the following set of equations and inequalities [16]:

$$
\left\{\begin{array}{c}
\boldsymbol{\varepsilon}^{p l E}=\mathbf{N}^{E} \boldsymbol{\lambda}^{E} \\
\boldsymbol{\Phi}^{E}=\left(\mathbf{N}^{E}\right)^{T} \boldsymbol{\sigma}-\mathbf{H}^{E} \boldsymbol{\lambda}^{E} \\
\boldsymbol{\Phi}^{E} \leq 0 \quad \boldsymbol{\lambda}^{E} \geq \mathbf{0} \\
\boldsymbol{\lambda}^{E} \boldsymbol{\Phi}^{E}=0
\end{array}\right.
$$

where, in the general context of a finite element discretization of the domain:

1. $\varepsilon^{p l E}$ is the plastic strain vector of the element $E$;

2. $\mathbf{N}^{E}$ is the shape functions matrix of the used finite element;

3. $\lambda^{E}$ is the plastic multiplier vector;

4. $\mathbf{H}^{E}$ is the hardening matrix, which in this case is diagonal and with non-null values, very small with the aim of reproducing the elastic-perfectly plastic case;

5. $\boldsymbol{\Phi}^{E}$ is a vector collecting the $r$ linearization planes of the failure surface.

6. $\boldsymbol{\sigma}$ is the vector of stress parameters that define point by point the stress (or internal actions) acting on the finite element.

Hypotheses assumed are: (1) the plasticity condition is piecewise-linearized with $r$ linearly elastic-plastic interacting planes in the space of superimposed stress and strain components; (2) unloading of yielded stress-points does not occur; (3) the continuum is discretized into constant strain and stress finite elements.

Alternatively [16] the solution of $(8)$ can be achieved using quadratic programming:

$$
\left\{\begin{array}{c}
\max \left\{-\frac{1}{2}\left(\lambda^{E}\right)^{T} \mathbf{H}^{E} \boldsymbol{\lambda}^{E}+\left(\lambda^{E}\right)^{T}\left(\mathbf{N}^{E}\right)^{T} \mathbf{D}^{E} \boldsymbol{\varepsilon}^{E}\right. \\
\text { subject to }: \boldsymbol{\lambda}^{E} \geq \mathbf{0}
\end{array}\right.
$$

where $\mathbf{D}^{E}$ is the elastic stiffness matrix, $\boldsymbol{\varepsilon}^{E}$ is the elastic part of the strain vector and all the other symbols have been already introduced.

The QP problem ( 9 ) is handled step by step on the incremental problem within an existing optimization code. Details of the numerical model, especially for what 
concerns the identification of the softening branch for the non-linear springs are given in [10][11] and are not repeated here for the sake of conciseness.
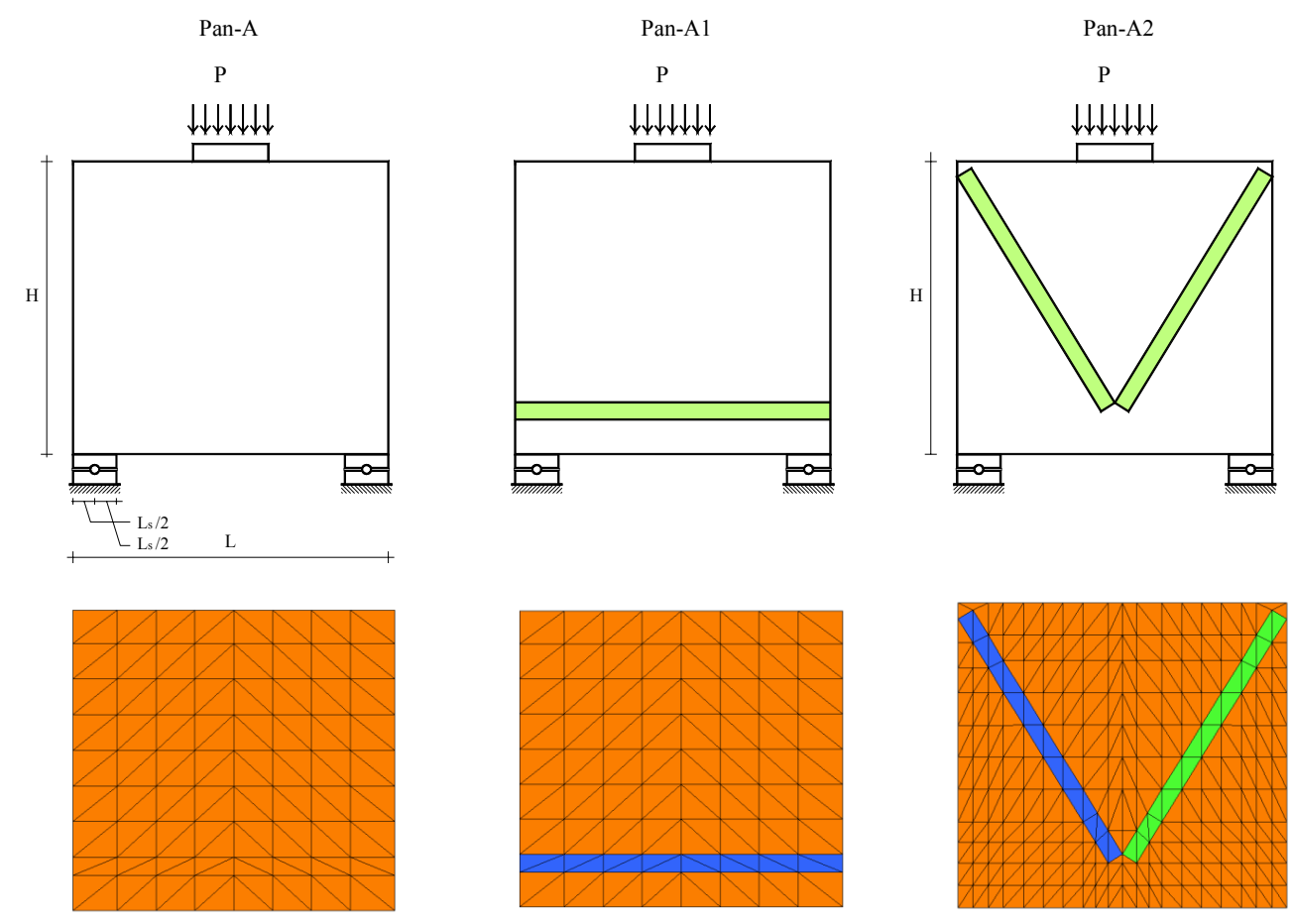

Figure 5: Masonry deep beam. Geometry, loading condition and FE discretization adopted for the numerical analyses.

\subsection{Numerical simulations}

Three masonry panels with and without CFRP strips strengthening, denoted as PANA, are here examined, see Figure 5. All panels, built with $1 / 4$ of common solid clay Italian bricks (dimensions $62.5 \times 30 \times 14 \mathrm{~mm}$ ), have dimensions $290 \times 270 \mathrm{~mm}$ (base $\times$ height). PAN-A is the non-strengthened wall, whereas PAN-A1 and PANA2 are specimens strengthened with different CFRP strip arrangements: a single horizontal strip for PAN-A1 and two symmetrical diagonal strips for PAN-A2. For these panels, several results are available [8]. The experimental tests were performed statically increasing the vertical external load applied at the top edge. The obtained results in terms of force-displacement diagrams (i.e. vertical load applied versus displacement of the steel plate that transfers the load to the panel) show key aspects induced by the CFRP strengthening on the global response of the panels. Furthermore, the examination of the crack paths during and after the tests shows important information on the effectiveness of the numerical model here proposed and on the contribution of the strengthening.

Mechanical properties of masonry panels were experimentally determined, as uniaxial compression tests were conducted on bricks, mortar and masonry specimens according to the indications of the Italian code of practice D.M. 
20/11/1987 [17]. The strengthening is constituted by high-strength carbon fiber sheets. FRP parameters adopted in the model have been deduced from experimental tests and from theoretical considerations, making use of CNR-DT200 0.
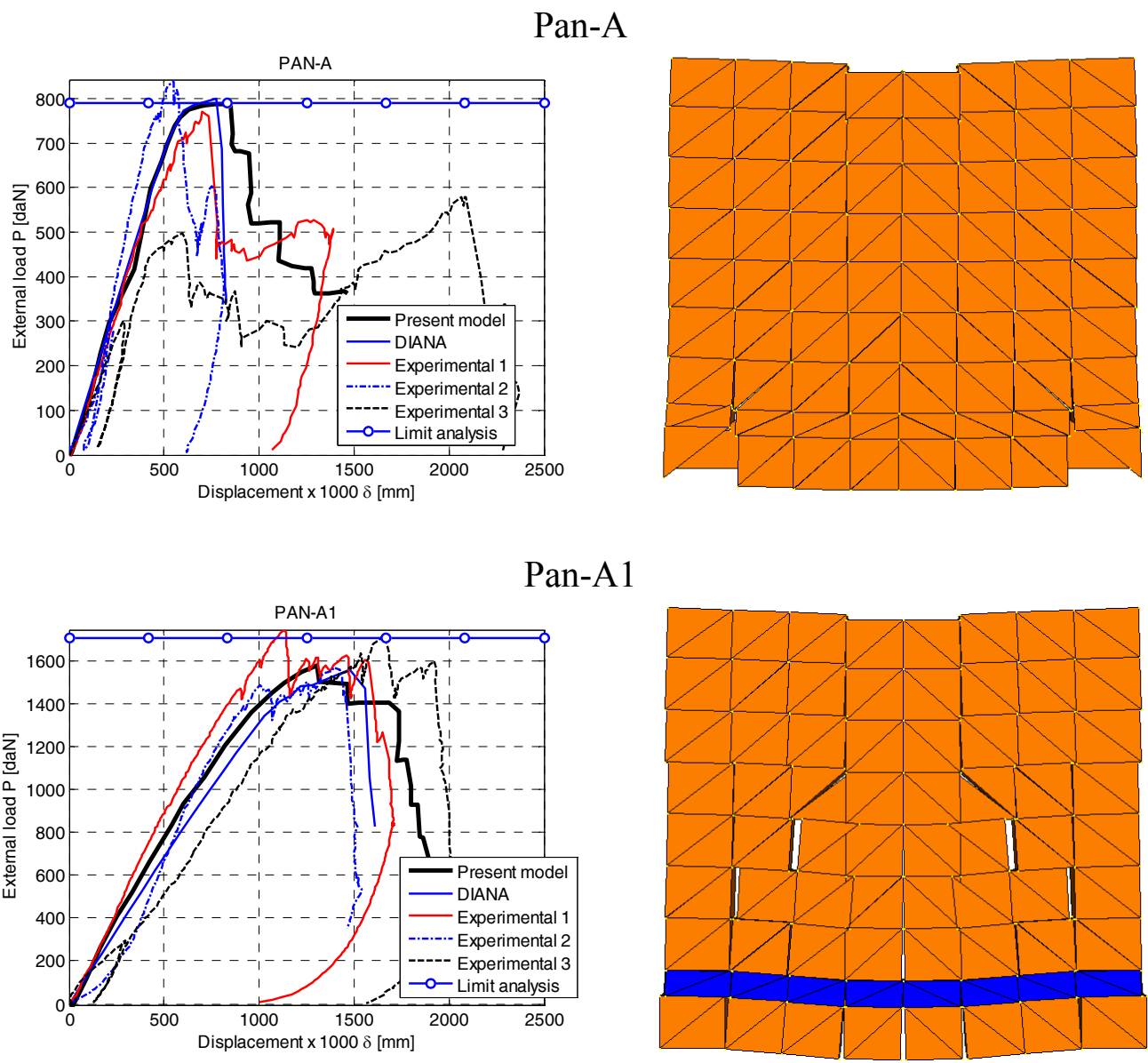

Pan-A2
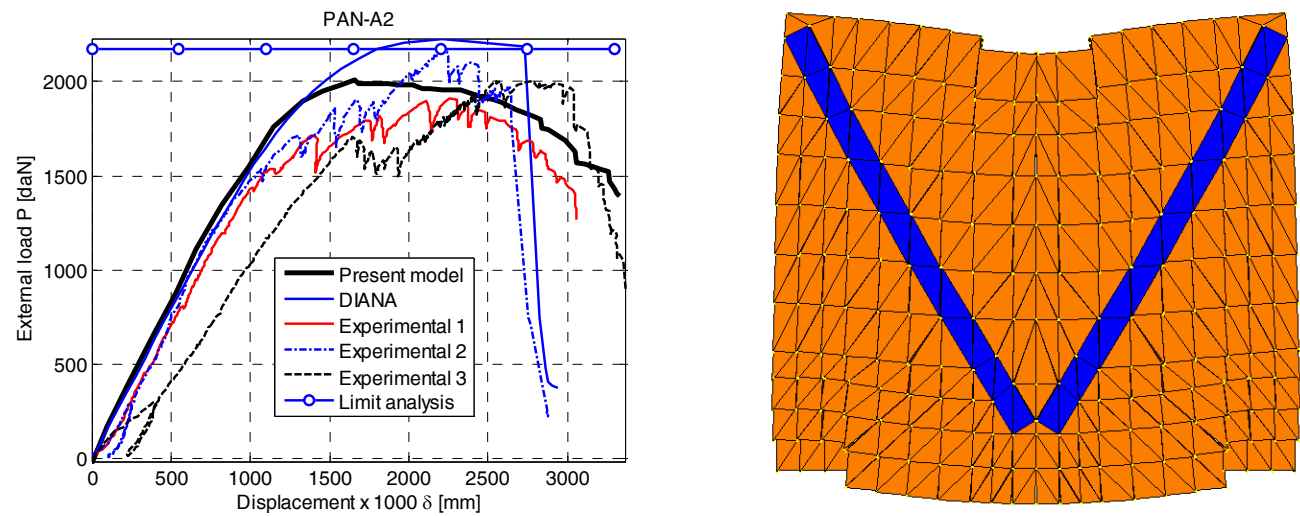

Figure 6: Masonry deep beam. Left: Comparison among load-displacement curves or collapse loads provided by experimentation, limit analysis and nonlinear FE codes (commercial and present software). Right: Deformed shapes at peak 
Since no information on the fracture energy and the post peak parameters for mortar were available, they are chosen according to the experimental results obtained by Van der Pluijm [18] on masonry specimens characterized by similar mechanical properties. The joints compressive strength $f_{c}$ adopted in the numerical simulations is assumed equal to the experimental masonry compressive strength value as all the non-linearity is concentrated on interfaces, see Table 1. For what concerns the mechanical parameters adopted for FRP/masonry triangular interfaces, a fracture energy equal to that evaluated using CNR DT-200 0 recommendations is adopted.

It is worth noting that, see Figure 5, all series were placed on steel plates of length Ls equal to $40 \mathrm{~mm}$, disposed at the lower edge extremes and positioned on steel rollers allowing rotation of the supports. The rotation of the lower edge extremes has minor effect on the numerical results [8] and is not considered here for the sake of simplicity.

Experimental load-displacement curves for the three series of panels here analyzed, see Figure 6, show that the introduction either of a horizontal strengthening (PAN-A1) or a double diagonal strengthening (PAN-A2) results in a considerable increase of the ultimate load.

\section{Pan-A}

Pan-A1

Normal stress
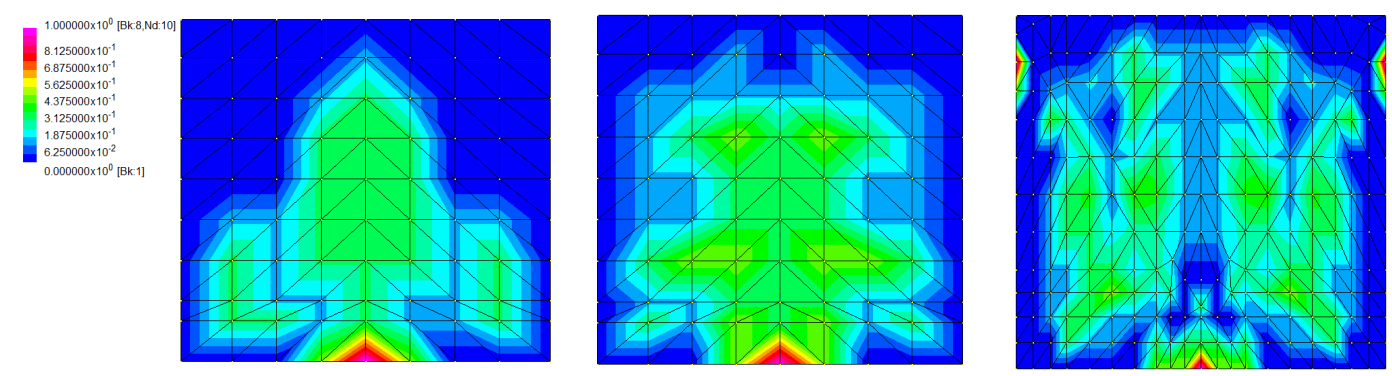

Shear
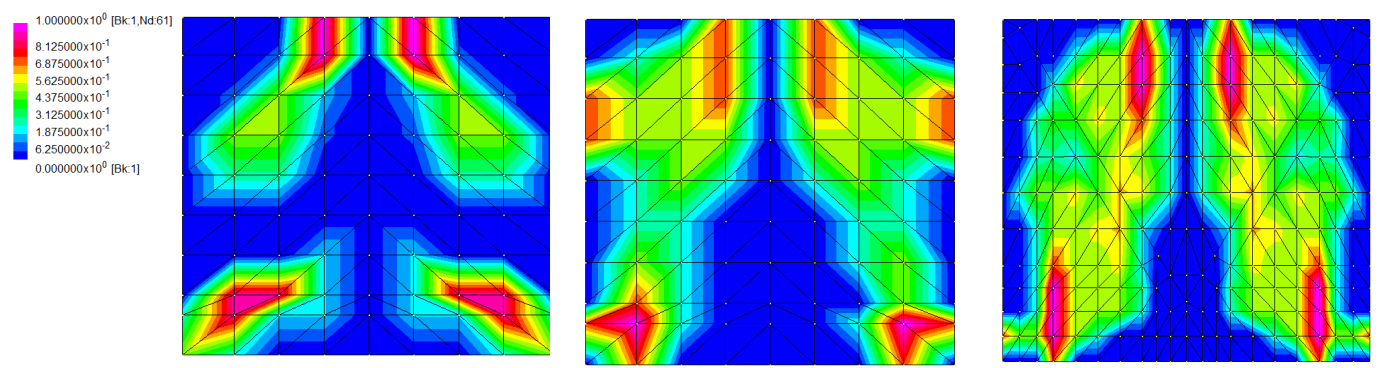

Figure 7: Masonry deep beam. Degraded interfaces patch for normal and shear stress (from 0 -no degradation- to 1 -full degradation) obtained through the nonlinear homogenized FE code proposed. 
In Figure 6, (i) the force-displacement curves of the point of application of the external load (center of the steel plate) from the two-step approach proposed, (ii) the ultimate load from an upper bound FE limit analysis software derived directly from the present one assuming interfaces rigid-plastic and (iii) the experimental forcedisplacement curves are reported for all the panels. Additionally, (iv), simulations performed with the commercial code DIANA [19], where an orthotropic elastoplastic with softening macroscopic model is adopted for masonry, are also represented to further assess present numerical results. Full details of the latter model may be found in [8].

For the un-strengthened panel (PAN-A), it is interesting to notice that the results obtained using the two-step approach here presented are, near the peak point, almost identical to experimental data, furnishing also a strength value in very good agreement with DIANA simulations. Also the initial stiffness and the post peak behaviour are reproduced very well.

For the strengthened panel PAN-A1, the present model exhibits a forcedisplacement curve in good agreement with both experimental data and commercial code DIANA simulations, also in the post-peak range. The results obtained for PAN-A2 are again very near to experimental ones, both in terms of peak-strength and post-peak behaviour. The acceptable differences between present model and DIANA may be explained remembering that within DIANA the strengthening is modelled by means of truss elements perfectly bonded to the masonry surface, where delamination is accounted for limiting tensile strength to a threshold value.
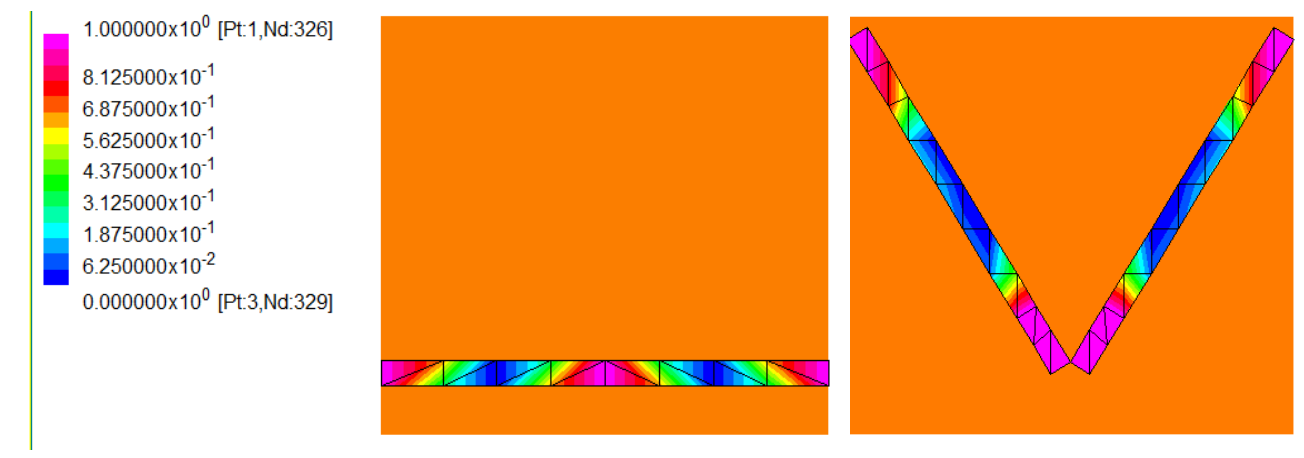

Figure 8: Masonry deep beam. Degraded FRP-masonry interfaces patch for shear action (from 0 -no degradation- to 1 -full degradation) obtained through the nonlinear homogenized FE code proposed (left: Pan-A1. Right: Pan-A2).

\section{References}

[1] CNR-DT 200-2004, "Guide for the design and construction of externally bonded FRP systems for strengthening existing structures", C.N.R. National Research Council, Italy, 2004.

[2] L. Gambarotta, S. Lagomarsino, "Damage models for the seismic response of brick masonry shear walls. Part II: The continuum model and its applications", Earthquake Engineering and Structural Dynamics 26(4): 441-462, 1997. 
[3] L. Berto, A. Saetta, R. Scotta, R. Vitaliani, “An orthotropic damage model for masonry structures", Int J Numer Methods Engng 55: 127-157, 2002.

[4] S. Brasile, R. Casciaro, G. Formica, "Finite Element formulation for nonlinear analysis of masonry walls", Computers \& Structures 88(3-4): 135-143, 2010.

[5] P.B. Lourenço, R. de Borst, J.G. Rots, "A plane stress softening plasticity model for orthotropic materials", International Journal for Numerical Methods in Engineering 40: 4033-4057, 1997.

[6] S. Pietruszczak, R. Ushaksarei, Failure criterion for structural masonry based on critical plane approach Journal of Engineering Mechanics ASCE 128(7): 769-778, 2002.

[7] S. Pietruszczak, R. Ushaksarei, "Description of inelastic behaviour of structural masonry", Int J Solids Struct 40: 4003-19, 2003.

[8] E. Grande, G. Milani, E. Sacco, "Modelling and analysis of FRP-strengthened masonry panels", Engineering Structures, 30 (7): 1842-1860, 2008.

[9] G. Milani, E. Milani, A. Tralli, "Upper Bound limit analysis model for FRPreinforced masonry curved structures. Part II: structural analyses", Computers \& Structures 87 (23-24): 1534-1558, 2009.

[10] G. Milani, A. Tralli, "Simple SQP approach for out-of-plane loaded homogenized brickwork panels accounting for softening", Computers \& Structures 89(1-2): 201-215, 2011.

[11] G. Milani, A. Tralli, "A simple meso-macro model based on SQP for the nonlinear analysis of masonry double curvature structures" International Journal of Solids and Structures 49(5): 808-834, 2012.

[12] S. Casolo, G. Milani, "A simplified homogenization-discrete element model for the non-linear static analysis of masonry walls out-of-plane loaded", Engineering Structures 32 (8): 2352-2366, 2010.

[13] P.B. Lourenço, J. Rots, J., "A multi-surface interface model for the analysis of masonry structures", Journal of Engineering Mechanics ASCE 123 (7): 660668, 1997.

[14] T. Massart, R.H.J., Peerlings, M.G.D. Geers, "Mesoscopic modeling of failure and damage-induced anisotropy in brick masonry", Eur J Mech A/Solids 23: 719-735, 2004.

[15] P. Pegon, A. Anthoine, "Numerical strategies for solving continuum damage problems with softening: application to the homogenisation of masonry", Computers \& Structures 64 (1-4): 623-642, 1997.

[16] O. De Donato, A. Franchi, "A modified gradient method for finite element elastoplastic analysis by quadratic programming", Computer Methods in Applied Mechanics and Engineering, 2(2): 107-131, 1973.

[17] D.M. 1987, "Norme tecniche per la progettazione, esecuzione e collaudo degli edifici in muratura e per il loro consolidamento", Italy, 1987.

[18] R. van der Pluijm, "Material properties of masonry and its components under tension and shear", in: Proc. 6th Canadian masonry symposium, 1992.

[19] DIANA 9.3 version, "User's Manual", TNO Building and Construction Research, Department of Computational Mechanics, Delft, The Netherlands, 2008. 